$\begin{array}{ll}\text { Research Square } & \begin{array}{l}\text { Preprints are preliminary reports that have not undergone peer review. } \\ \text { They should not be considered conclusive, used to inform clinical practice, } \\ \text { or referenced by the media as validated information. }\end{array}\end{array}$

\title{
Applying the RE-AIM implementation framework to evaluate fall prevention interventions in community dwelling adults with cognitive impairment: $A$ review
}

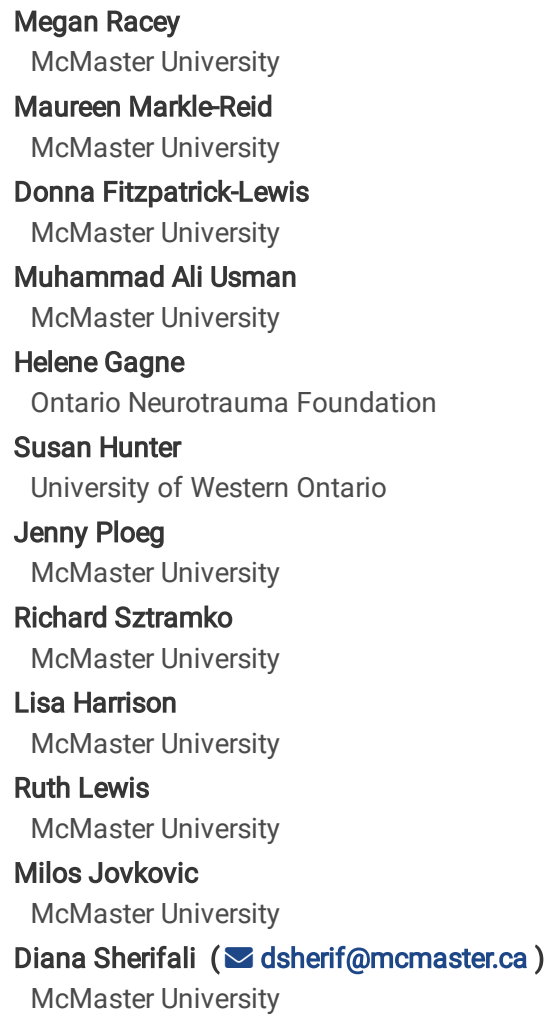

\section{Research Article}

Keywords: Fall Prevention, Cognitive Impairment, Older Adults, RE-AIM, Implementation

Posted Date: April 16th, 2021

DOI: https://doi.org/10.21203/rs.3.rs-395312/v1

License: () (i) This work is licensed under a Creative Commons Attribution 4.0 International License. Read Full License

Version of Record: A version of this preprint was published at BMC Geriatrics on July 26th, 2021. See the published version at https://doi.org/10.1186/s12877021-02376-7. 


\section{Abstract}

Background: Cognitive impairment $(\mathrm{Cl})$ is a risk factor for falls due to environmental or living settings, balance, gait and vision impairments, as well as medications. While previous systematic reviews have focused on the effectiveness of fall prevention programs in adults with cognitive impairment (1-4), but very limited information is available on their implementation. This review examines what aspects of fall prevention interventions for community-dwelling adults with $\mathrm{Cl}$ have been reported using the Reach, Effectiveness, Adoption, Implementation, and Maintenance (RE-AIM) framework to support successful implementation.

Methods: We examined the included studies from our systematic review, which searched 7 databases for fall prevention interventions involving communitydwelling adults $\geq 50$ years with mild to moderate Cl. Reviewers screened citations and extracted data for study characteristics and the 5 dimensions ( 62 criteria) of the RE-AIM framework.

Results: 12 randomized controlled trials (RCTs) consisting of 8 exercise interventions, 3 multifactorial interventions, and 1 medication treatment were included in the review. Only 4 of 62 criteria were reported by all 12 included studies and 29 criteria were not reported by any of the studies. Five of the included studies reported on 20 or more of the 62 possible RE-AIM criteria and three of these studies were ones that self-identified as "feasibility" studies. While Reach was the best-reported construct by the included studies, followed by Effectiveness and Implementation, the criteria within the Adoption and Maintenance constructs were rarely mentioned by these studies. In general, there was also wide variation in how each of the criteria were reported on by study authors

Conclusion: Based on the reporting of RE-AIM components in this review, we are unable to make connections to successful intervention components and thus practice-based recommendations for fall prevention in those with $\mathrm{Cl}$. The lack of detail regarding implementation approaches greatly limits the interpretation and comparisons across studies to fully understand inform future research efforts.

\section{Background}

Falls result in adverse outcomes for both individuals and their caregivers and families due to injuries, health complications, and decreased quality of life (16). Falls also cost our public health system as they are the leading cause of injury-related admissions to acute care hospitals and in-hospital deaths. With an aging population the cost of fall injuries to seniors in Canada is estimated to rise from \$2.4 billion a year in direct healthcare costs (7) to $\$ 240$ billion by 2040 (8).

Cognitive impairment is a well-recognized risk factor for falls due to multifactorial reasons, including the environment or living settings, balance, gait and vision impairments, as well as medications leading to an increased state of confusion $(3,9,10)$. Older adults with dementia fall two to three times more than cognitively healthy older adults (11) and $60-80 \%$ of people with dementia fall annually (3). However, the risk factors for falls in adults with cognitive impairment are unique (12), limiting the ability of practitioners and clinicians to take what is known regarding fall prevention in cognitively healthy individuals (13-17) and translate these findings to patients with cognitive impairment (18). The result is that little evidence is available to clinicians and health practitioners to guide practice to reduce falls in community-dwelling adults with cognitive impairment, specifically.

While it has been recognized that fall prevention interventions need to address multifactorial intrinsic and extrinsic risk factors and the body of evidence is growing in this population (3), the applicability and implementation of such fall prevention interventions to older adults with cognitive impairment is undetermined. Previous systematic reviews have focused on the effectiveness of fall prevention programs in adults with cognitive impairment (1-4), but very limited information is available on their implementation. This gap in the research for reducing falls in those with cognitive impairment and the need for more detailed reporting of these interventions has been recognized previously (3) and still goes unmet. Knowledge of the factors that influence the implementation of fall prevention interventions may enhance their adoption and use (19).

The Reach, Effectiveness, Adoption, Implementation, and Maintenance (RE-AIM) framework was developed to improve the reporting of essential program elements that can advance the sustainable adoption and implementation of effective, generalizable, evidence-based interventions (20). While the RE-AIM framework has been used in the planning, translation, dissemination, and evaluation of individual fall prevention interventions for older adults (21-23), to date, no fall prevention systematic review has summarized the proportion of studies which report on various RE-AIM dimensions or components. While many implementation theories exist, the advantage of using the RE-AIM framework is that it provides a framework for relevant intervention outcomes, such as the effectiveness of the intervention and their implementation strategies, but also on the potential for further application, adoption, and scaling of these interventions for long-term maintenance of successful programs. This, in turn, will help individuals to select effective interventions with potential for broader impact.

This paper builds on a related systematic review in which we investigated the effectiveness of fall prevention interventions in community-dwelling adults with mild to moderate cognitive impairment (24). In this paper, we examine the application and reporting of various dimensions of the RE-AIM framework in the studies included in the systematic review to inform future practice-based implementation research of such fall prevention initiatives.

\section{Methods}

This review is a secondary research question to a systematic review and meta-analysis (22). This paper examines the included studies from our systematic review of the fall prevention literature in community-dwelling adults diagnosed with mild to moderate cognitive impairment (24) from the registered protocol (PROSPERO-CRD42020210916).

\section{Search Strategy}


The search terms, databases, and strategy were developed in consultation with a research librarian at McMaster University and informed by previous systematic reviews $(4,6,25)$ (Additional File 1). We searched MEDLINE, Embase, PsycINFO, Cochrane Central Register of Controlled Trials (CENTRAL), Cumulative Index of Nursing and Allied Health Literature (CINAHL), Web of Science, and Science Direct up to April 2020 and manually searched reference lists of relevant reviews and included studies for citations not captured in our search. Results from the search were deduplicated, and citations were uploaded to a secure internet-based platform for screening (DistillerSR, Evidence Partners Inc., Ottawa, Canada).

\section{Eligibility Criteria}

The eligibility criteria were established for the primary research question from the original review (24) and have been previously explained in detail. Briefly, we included fall prevention interventions in community-dwelling adults (aged 50+) with mild and/or moderate cognitive impairment (Cl). $\mathrm{Cl}$ had to be assessed by a valid and reliable tool, diagnosis, or medical report, and/or clearly identified and described by study authors. For our review, community dwelling included individuals living in a community setting (with or without caregiver support) and can include different locations/settings, however, we excluded those living in retirement homes, nursing homes, long-term care homes, acute care, or hospital settings where they may receive full-time support and care for activities of daily living. We also excluded studies of older adults with severe $\mathrm{Cl}$.

The main purpose of the intervention had to be either primary or secondary fall prevention as defined by the Institute for Work and Health (26) Studies must have been available in English, peer-reviewed, and comprised of interventions with a control group (randomization was not required). For our review, a control group was defined as treatment as usual, usual care (i.e., no change to usual activities), or minimal contact (an intervention not thought to reduce falls such as general health education or social visits). We did not include or exclude studies based on outcomes measured.

\section{Data Extraction}

A team of researchers conducted the screening and data extraction (M.R., D.F.L., R.L., M.J.). A minimum of two reviewers were required to independently and in duplicate screen titles and abstracts of all potentially eligible studies. Articles marked for inclusion by either team member went on to full-text screening which was completed independently and in duplicate by 2 team members and required consensus for inclusion or exclusion. We developed, piloted, and deployed standardized forms for data extraction. Two team members independently completed full data extraction of study characteristics (setting, sample size, inclusion and exclusion criteria, characteristics of participants, type of intervention (categories based on existing literature (27) and taxonomies (28)), and experimental and control components) and the 5 dimensions (62 criteria) of RE-AIM.

For the RE-AIM data extraction, reviewers used an adapted extraction tool designed specifically for conducting systematic reviews using RE-AIM (29). Reach was evaluated by 12 criteria including descriptions of the target population, inclusion, and exclusion criteria, who participated or was exposed to the intervention, participation rates, and characteristics of those who participated and those who did not. Effectiveness (or efficacy) was evaluated by 9 RE-AIM criteria including reporting of mediators and moderators, how data were treated, quality of life, unintended or negative consequences, and attrition. Adoption was assessed at both the setting and provider/staff levels by 10 and 11 criteria, respectively. The Adoption construct included criteria such as the number and proportion of setting and staff members who agreed to participate in delivering the intervention, description of target locations or providers, how these settings and staff members were recruited, and how representative they were of the intended audience in terms of setting and staff. Implementation was assessed by

11 criteria as our research team added 2 criteria (engagement to inform intervention development and tailoring of intervention). Other existing criteria included whether interventions were theory-based, detailed descriptions of intervention protocols and how well these protocols were adhered to (fidelity), costs, and the completion rates of intervention participants. Maintenance was evaluated by 9 RE-AlM criteria including sustained impact of the intervention after termination for the participants and at the setting/staff level.

We included two additional components from the template for intervention description and replication (TIDieR) checklist and guide (30), as these are not covered by RE-AIM: details about intervention tailoring and engagement of practitioners, participants, and/or caregivers in the development of the intervention. We also considered patient experience, caregiver outcomes and provider experience in extraction as additional data of interest. See Additional File 2 for REAIM components and definitions of each criteria. The lead researcher of this review (M. R.) resolved conflicts.

\section{Results}

From 20,727 citations, we assessed 479 full-text articles for eligibility, and included 12 randomized controlled trials in this review (Fig. 1) (31). The majority of studies $(n=8)$ were exercise interventions $(31-38)$, while 3 were multifactorial $(5,39,40)$, and 1 provided medication treatment $(41)$. Five of the 12 included studies were self-described as "feasibility" studies $(5,31,32,36,37)$. Overall, the studies were published from 2010 to 2020 . Characteristics of the included studies can be found in Table 1 and further demographic data from studies can be found in Additional File 3. A total sample of 509 community-dwelling adults with mild to moderate cognitive impairment were included in this review with a mean age ranging from 67.5 to 84.0 years and percentage of women in the studies ranging from $20-74 \%$. All included studies had fewer than 122 participants with most studies consisting of less than 50 participants total ( $\mathrm{n}=8$ ). Studies were conducted across the globe in North America, South America, Europe, Asia, and Australia, and intervention duration was between 4 weeks to one year, but most studies $(n=8)$ were between 12 weeks and 6 months in duration. While all participants were community-dwelling, interventions took place in a combination of settings including home $(n=6)$, community centres $(n=3)$, and research centres $(n=2)$. 
Table 1

Fall Prevention Study Details

\begin{tabular}{|c|c|c|c|c|c|c|c|c|c|}
\hline Study, Year & $\mathbf{N}^{1}$ & $\begin{array}{l}\text { Age, } \\
\text { mean } \\
\text { y (SD) }\end{array}$ & $\begin{array}{l}\text { Gender }^{2} \\
(\mathrm{~F} / \mathrm{M}, \\
\%)\end{array}$ & $\begin{array}{l}\text { Cognitive } \\
\text { Impairment } \\
\text { Tool \& } \\
\text { Baseline } \\
\text { Score }\end{array}$ & $\begin{array}{l}\text { Study } \\
\text { Design }\end{array}$ & $\begin{array}{l}\text { Intervention } \\
\text { Duration }\end{array}$ & $\begin{array}{l}\text { Intervention } \\
\text { Category \& Setting }\end{array}$ & Control & Harms \\
\hline \multirow[t]{2}{*}{$\begin{array}{l}\text { Varriano, } \\
2020\end{array}$} & \multirow[t]{2}{*}{7} & \multirow{2}{*}{$\begin{array}{l}\text { 0: } \\
79.1 \\
(6.7)\end{array}$} & \multirow[t]{2}{*}{$57 / 43$} & MoCA & \multirow[t]{2}{*}{$\mathrm{RCT}$} & \multirow[t]{2}{*}{12 weeks } & \multirow{2}{*}{$\begin{array}{l}\text { Exercise; vestibular } \\
\text { exercises } \\
\text { N/R }\end{array}$} & \multirow[t]{2}{*}{ Usual care } & \multirow[t]{2}{*}{$\begin{array}{l}\text { Falls, but unclear if due to } \\
\text { intervention }\end{array}$} \\
\hline & & & & $\begin{array}{l}\text { O: } 21.2 \\
(2.9)\end{array}$ & & & & & \\
\hline \multirow{4}{*}{$\begin{array}{l}\text { Goldberg, } \\
2019\end{array}$} & \multirow[t]{4}{*}{60} & \multirow{4}{*}{$\begin{array}{l}\text { O: } 76 \\
\text { (range } \\
65- \\
91 \text { ) }\end{array}$} & \multirow[t]{4}{*}{$43 / 57$} & & \multirow[t]{4}{*}{$\mathrm{RCT}$} & \multirow[t]{4}{*}{12 months } & \multirow{2}{*}{$\begin{array}{l}\text { Exercise; Balance, } \\
\text { strength, dual-task } \\
\text { training, gait re- } \\
\text { education }\end{array}$} & \multirow{4}{*}{$\begin{array}{l}\text { Single falls } \\
\text { prevention } \\
\text { assessment }\end{array}$} & \multirow{4}{*}{$\begin{array}{l}19 \text { recorded adverse events } \\
\text { ( } 5 \text { non-serious but } \\
\text { intervention related })\end{array}$} \\
\hline & & & & $\begin{array}{l}\text { O: } 25.6 \\
(3.1)\end{array}$ & & & & & \\
\hline & & & & $\begin{array}{l}\text { I: } 24.8 \\
(3.6) ; 26.2 \\
(3.2) ;\end{array}$ & & & \multirow[t]{2}{*}{ Home-based } & & \\
\hline & & & & $\begin{array}{l}\text { C: } 25.9 \\
(2.4)\end{array}$ & & & & & \\
\hline \multirow[t]{4}{*}{$\begin{array}{l}\text { Padala, } \\
2017\end{array}$} & \multirow[t]{4}{*}{30} & \multirow{2}{*}{$\begin{array}{l}0: \\
73.0 \\
(6.2)\end{array}$} & \multirow[t]{4}{*}{$37 / 63$} & MMSE & \multirow[t]{4}{*}{ RCT } & \multirow[t]{4}{*}{8 weeks } & \multirow{4}{*}{$\begin{array}{l}\text { Exercise; Wii-fit } \\
\text { (yoga, strength, } \\
\text { aerobics, balance) } \\
\text { Home-based }\end{array}$} & \multirow{4}{*}{$\begin{array}{l}\text { Self-paced } \\
\text { walking } \\
\text { program }\end{array}$} & \multirow[t]{4}{*}{ None study related } \\
\hline & & & & $\begin{array}{l}\text { O: } 22.9 \\
(2.2)\end{array}$ & & & & & \\
\hline & & $\begin{array}{l}\text { I: } 72.1 \\
(5.3) ;\end{array}$ & & $\begin{array}{l}\text { I: } 23.3 \\
(2.2)\end{array}$ & & & & & \\
\hline & & $\begin{array}{l}\text { C: } \\
73.9 \\
(7.1)\end{array}$ & & $\begin{array}{l}\text { C: } 22.7 \\
(2.3)\end{array}$ & & & & & \\
\hline \multirow[t]{4}{*}{$\begin{array}{l}\text { Zieschang, } \\
2017\end{array}$} & \multirow[t]{4}{*}{122} & \multirow{4}{*}{$\begin{array}{l}\text { I: } 82.1 \\
(6.6) ; \\
\text { C: } \\
82.2 \\
(6.7)\end{array}$} & \multirow[t]{4}{*}{$74 / 26^{2}$} & & \multirow[t]{4}{*}{$\mathrm{RCT}$} & 3 months & $\begin{array}{l}\text { Exercise; progressive } \\
\text { resistance and }\end{array}$ & $\begin{array}{l}\text { Seated motor } \\
\text { training }\end{array}$ & $\mathrm{N} / \mathrm{R}$ \\
\hline & & & & $\begin{array}{l}\text { I: } 21.6 \\
(2.9)\end{array}$ & & & $\begin{array}{l}\text { functional training } \\
\text { (activities of daily } \\
\text { living, balance, }\end{array}$ & exercises & \\
\hline & & & & $\begin{array}{l}\text { C: } 21.9 \\
(3.3)\end{array}$ & & & walking, gait) & & \\
\hline & & & & & & & $\mathrm{N} / \mathrm{R}$ & & \\
\hline $\begin{array}{l}\text { Sungkarat, } \\
2017\end{array}$ & 66 & $\begin{array}{l}\text { I: } 68.3 \\
(6.7) ;\end{array}$ & $50 / 50$ & $\begin{array}{l}\text { MoCA, } \\
\text { MMSE }\end{array}$ & $\mathrm{RCT}$ & 15 weeks & Exercise; Tai Chi & $\begin{array}{l}\text { Educational } \\
\text { material }\end{array}$ & No adverse events found. \\
\hline & & $\begin{array}{l}\text { C: } \\
67.5 \\
(7.3)\end{array}$ & & $\begin{array}{l}\text { I: MoCA: } \\
21.2 \text { (3.4), } \\
\text { MMSE: } \\
26.5(1.7)\end{array}$ & & & $\begin{array}{l}\text { Community-centre } \\
\text { and home-based }\end{array}$ & $\begin{array}{l}\text { covering } \\
\text { information } \\
\text { related to } \\
\text { cognitive } \\
\text { impairment } \\
\text { and fall }\end{array}$ & \\
\hline & & & & $\begin{array}{l}\text { C: MoCA: } \\
20.4 \text { (3.8), } \\
\text { MMSE: } \\
25.8 \text { (2.3) }\end{array}$ & & & & prevention & \\
\hline $\begin{array}{l}\text { Schwenk, } \\
2016\end{array}$ & 22 & 0: & $55 / 45$ & MoCA & RCT & 4 weeks & $\begin{array}{l}\text { Exercise; Balance } \\
\text { (ankle point-to-point }\end{array}$ & Usual care & No training-related adverse \\
\hline & & & & $\begin{array}{l}\text { O: } 23.3 \\
(2.6) ;\end{array}$ & & & $\begin{array}{l}\text { reaching tasks and } \\
\text { virtual obstacle- }\end{array}$ & & \\
\hline & & $\begin{array}{l}\text { l: } 77.8 \\
(6.9)\end{array}$ & & I: 23.3 & & & crossing tasks) & & \\
\hline & & C: & & (3.1); & & & Research centre & & \\
\hline & & $\begin{array}{l}79.0 \\
(10.4)\end{array}$ & & $\begin{array}{l}\text { C: } 22.4 \\
(3.0)\end{array}$ & & & & & \\
\hline
\end{tabular}

$\mathrm{O}=$ overall population; $\mathrm{I}=$ intervention; $\mathrm{C}=$ control; $\mathrm{N} / \mathrm{R}=$ not reported; $\mathrm{RCT}=$ randomized controlled trial; $\mathrm{CCT}=$ clinical (non-randomized) controlled trial. MoCA = Montreal cognitive assessment; $M M S E$ = Mini Mental State Exam; ACE-R = Addenbrooke's cognitive examination - revised; CDR = Clinical Dementia Rating scale; TUG = timed up and go test; $C S T$ = chair sit stand test; FRT = functional reach test

1. Number of participants randomized to intervention; 2 . Values for gender are based on reported baseline which may not equal $\mathrm{N}$ randomized but rather the number of participants who completed the intervention; 3 . Not including follow-up, if applicable. 


\begin{tabular}{|c|c|c|c|c|c|c|c|c|c|}
\hline Study, Year & $\mathbf{N}^{1}$ & $\begin{array}{l}\text { Age, } \\
\text { mean } \\
\text { y (SD) }\end{array}$ & $\begin{array}{l}\text { Gender }^{2} \\
(\mathrm{~F} / \mathrm{M} \text {, } \\
\%)\end{array}$ & $\begin{array}{l}\text { Cognitive } \\
\text { Impairment } \\
\text { Tool \& } \\
\text { Baseline } \\
\text { Score }\end{array}$ & $\begin{array}{l}\text { Study } \\
\text { Design }\end{array}$ & $\begin{array}{l}\text { Intervention } \\
\text { Duration }^{3}\end{array}$ & $\begin{array}{l}\text { Intervention } \\
\text { Category \& Setting }\end{array}$ & Control & Harms \\
\hline $\begin{array}{l}\text { Montero- } \\
\text { Odasso, } \\
2019\end{array}$ & 60 & $\begin{array}{l}\text { O: } \\
75.28 \\
\text { (7.18); } \\
\text { I: } \\
73.45 \\
(5.74) ; \\
\text { C: } \\
77.24 \\
(8.11)\end{array}$ & $45 / 55$ & $\begin{array}{l}\text { MMSE, } \\
\text { MoCA } \\
\text { O: sMMSE: } \\
27.47 \\
\text { (1.96), } \\
\text { MoCA: } \\
23.60 \\
(2.52) \text {; } \\
\text { l: sMMSE: } \\
27.42 \\
(2.19) \\
\text { MoCA: } \\
23.19 \\
(2.55) ; \\
\text { C: sMMSE: } \\
27.52 \\
\text { (1.72), } \\
\text { MoCA: } \\
22.97 \\
\text { (2.37) }\end{array}$ & RCT & 6 months & $\begin{array}{l}\text { Medication or } \\
\text { vitamin supplement; } \\
\text { Donepezil } \\
\text { Home-based }\end{array}$ & Placebo & $\begin{array}{l}\text { No major adverse events } \\
\text { requiring treatment were } \\
\text { reported. }\end{array}$ \\
\hline Chen, 2018 & 30 & $\begin{array}{l}\text { I: } 77.3 \\
(9.4) \\
\text { C: } \\
77.3 \\
(10.0)\end{array}$ & $50 / 50$ & $\begin{array}{l}\text { MMSE, } \\
\text { CDR } \\
\text { I: MMSE: } \\
\text { 16.4 (7.3), } \\
\text { CDR: } 0.5= \\
6,1.0=6, \\
2.0=3 ; \\
\text { C: MMSE: } \\
\text { 17.9 (3.7), } \\
\text { CDR: } 0.5= \\
3,1.0=9, \\
2.0=1\end{array}$ & $\mathrm{RCT}$ & 2 months & $\begin{array}{l}\text { Multifactorial; } \\
\text { Musical dual-task } \\
\text { training (physical } \\
\text { and cognitive tasks) } \\
\text { Community/research } \\
\text { centre }\end{array}$ & $\begin{array}{l}\text { Non-musical } \\
\text { cognitive } \\
\text { tasks and } \\
\text { walking } \\
\text { exercises }\end{array}$ & No adverse events reported. \\
\hline Kim, 2017 & 30 & $\begin{array}{l}\text { I: } 82.0 \\
(4.6) \\
\text { C: } \\
80.9 \\
(3.4)\end{array}$ & $20 / 80$ & $\begin{array}{l}\text { MMSE- } \\
\text { Korea } \\
\text { I: } 15.5 \\
(2.9) ; \\
\text { C: } 15.6 \\
(2.4)\end{array}$ & CCT & 12 weeks & $\begin{array}{l}\text { Multifactorial; } \\
\text { physical activities, } \\
\text { cognitive activities, } \\
\text { activities of daily } \\
\text { living, music } \\
\text { activities } \\
\text { Community centre }\end{array}$ & Usual care & $\mathrm{N} / \mathrm{R}$ \\
\hline $\begin{array}{l}\text { Wesson, } \\
2013\end{array}$ & 22 & $\begin{array}{l}\text { I: } 78.7 \\
(4.2) \\
\text { C: } \\
80.9 \\
(5.0)\end{array}$ & $41 / 59$ & $\begin{array}{l}\text { ACE-R, } \\
\text { MMSE } \\
\text { I: ACE-R: } \\
67.8(12.6), \\
\text { MMSE: } \\
24.5(3.1) ; \\
\text { C: ACE-R: } \\
62.5(14.2), \\
\text { MMSE: } \\
22.5(4.3)\end{array}$ & $\mathrm{RCT}$ & 12 weeks & $\begin{array}{l}\text { Multifactorial; } \\
\text { strength and balance } \\
\text { exercises, home } \\
\text { hazard reduction } \\
\text { Home-based }\end{array}$ & $\begin{array}{l}\text { Usual care, } \\
\text { health } \\
\text { promotion } \\
\text { brochures on } \\
\text { fall prevention } \\
\text { and home } \\
\text { safety }\end{array}$ & $\begin{array}{l}\text { No serious adverse events } \\
\text { related to the intervention } \\
\text { were reported. Minor } \\
\text { complaints relating to } \\
\text { stiffness, dizziness and } \\
\text { mild joint pain }(n=4 ; 36 \%) \\
\text { were reported. }\end{array}$ \\
\hline $\begin{array}{l}\text { Suttanon, } \\
2013\end{array}$ & 40 & $\begin{array}{l}\text { O: } \\
81.90 \\
\text { (5.72); } \\
\text { l: } \\
83.42 \\
(5.10) ; \\
\text { C: } \\
80.52 \\
(6.01)\end{array}$ & $63 / 37$ & $\begin{array}{l}\text { MMSE } \\
\text { I: } 20.89 \\
(4.74) ; \\
\text { C: } 21.67 \\
(4.43)\end{array}$ & $\mathrm{RCT}$ & 6 months & $\begin{array}{l}\text { Exercise; balance } \\
\text { and strength } \\
\text { exercises, walking } \\
\text { program } \\
\text { Home-based }\end{array}$ & $\begin{array}{l}\text { Education and } \\
\text { information } \\
\text { sessions on } \\
\text { the topic of } \\
\text { dementia and } \\
\text { ageing }\end{array}$ & $\begin{array}{l}\text { There were no falls or other } \\
\text { serious adverse events } \\
\text { associated with the } \\
\text { intervention }\end{array}$ \\
\hline
\end{tabular}

$\mathrm{O}=$ overall population; $\mathrm{I}$ = intervention; $\mathrm{C}=$ control; $\mathrm{N} / \mathrm{R}=$ not reported; $\mathrm{RCT}$ = randomized controlled trial; $\mathrm{CCT}=$ clinical (non-randomized) controlled trial. MoCA = Montreal cognitive assessment; MMSE = Mini Mental State Exam; ACE-R = Addenbrooke's cognitive examination - revised; CDR = Clinical Dementia Rating scale; TUG = timed up and go test; $C S T$ = chair sit stand test; FRT = functional reach test

1. Number of participants randomized to intervention; 2 . Values for gender are based on reported baseline which may not equal $\mathrm{N}$ randomized but rather the number of participants who completed the intervention; 3 . Not including follow-up, if applicable. 


\begin{tabular}{|c|c|c|c|c|c|c|c|c|c|}
\hline Study, Year & $\mathbf{N}^{1}$ & $\begin{array}{l}\text { Age, } \\
\text { mean } \\
\text { y (SD) }\end{array}$ & $\begin{array}{l}\text { Gender }^{2} \\
\text { (F/M, } \\
\%)\end{array}$ & $\begin{array}{l}\text { Cognitive } \\
\text { Impairment } \\
\text { Tool \& } \\
\text { Baseline } \\
\text { Score }\end{array}$ & $\begin{array}{l}\text { Study } \\
\text { Design }\end{array}$ & $\begin{array}{l}\text { Intervention } \\
\text { Duration }\end{array}$ & $\begin{array}{l}\text { Intervention } \\
\text { Category \& Setting }\end{array}$ & Control & Harms \\
\hline $\begin{array}{l}\text { Hernandez, } \\
2010\end{array}$ & 20 & $\begin{array}{l} \\
\\
\\
\\
\\
\text { I: } 77.7 \\
(7.6) ; \\
\text { C: } \\
84.0 \\
(6.1)\end{array}$ & N/R & $\begin{array}{l}\text { MMSE } \\
\text { I: } 16.4 \\
(6.7) ; \\
\text { C: } 14.2 \\
(5.1)\end{array}$ & СCT & 6 months & $\begin{array}{l}\text { Exercise; stretching, } \\
\text { weight training, } \\
\text { circuits, dance, } \\
\text { recreational } \\
\text { activities, relaxation } \\
\text { N/R }\end{array}$ & Usual care & N/R \\
\hline $\begin{array}{l}\mathrm{O}=\text { overall } \mathrm{p} \\
\text { MoCA = Mor } \\
\text { Dementia Re }\end{array}$ & $\begin{array}{l}\text { ulat } \\
\text { cal c } \\
\text { ig s }\end{array}$ & $\begin{array}{l}; I=\text { int } \\
\text { nitive a } \\
\text { e; TUG }\end{array}$ & $\begin{array}{l}\text { ention; } \mathrm{C}= \\
\text { essment; } \\
\text { med up al }\end{array}$ & $\begin{array}{l}\text { ontrol; } N / R=r \\
\text { MSE = Mini M } \\
\text { go test; CST }\end{array}$ & $\begin{array}{l}\text { ot reporte } \\
\text { ental State } \\
=\text { chair sit }\end{array}$ & $\begin{array}{l}\mathrm{d} ; \mathrm{RCT}=\text { randc } \\
\text { Exam; ACE-R } \\
\text { stand test; FR }\end{array}$ & $\begin{array}{l}\text { ized controlled trial; CC } \\
\text { Addenbrooke's cognitis } \\
\text { = functional reach test }\end{array}$ & $\begin{array}{l}=\text { clinical }(\mathrm{n} \\
\text { xamination }\end{array}$ & $\begin{array}{l}\text { ndomized) controlled trial. } \\
\text { vised; CDR = Clinical }\end{array}$ \\
\hline
\end{tabular}

\section{Overall RE-AIM Summary}

A summary of the RE-AIM results by each element can be found in Table 2 (detailed results are available in Additional File 4). Each study reported on at least one of the 62 RE-AIM criteria; only 4 criteria were reported by all 12 included studies and 29 criteria were not reported by any of the studies. The 4 criteria reported by all studies were participant characteristics, sample size, and intervention details, consistent with CONSORT guidelines (42). Five of the included studies reported on 20 or more of the 62 possible RE-AIM criteria $(5,32,33,37,39)$ and three of these studies were ones that self-identified as "feasibility" studies $(5,32,37)$. The study that reported the most criteria (23 out of 62 ) was a randomized trial to test the feasibility of study components and acceptability of a home hazard reduction and balance and strength exercise fall prevention program (5). 
Table 2

RE-AIM Criteria Included in Each Study

\begin{tabular}{|c|c|c|c|c|c|c|c|c|c|c|c|}
\hline & & Study & & & & & & & & & \\
\hline $\begin{array}{l}\text { RE-AIM } \\
\text { Element }\end{array}$ & Criteria & $\begin{array}{l}\text { Varriano, } \\
2020\end{array}$ & $\begin{array}{l}\text { Goldberg, } \\
2019\end{array}$ & $\begin{array}{l}\text { Padala, } \\
2017\end{array}$ & $\begin{array}{l}\text { Zieschang, } \\
2017\end{array}$ & $\begin{array}{l}\text { Sungkarat, } \\
2017\end{array}$ & $\begin{array}{l}\text { Schwenk, } \\
2016\end{array}$ & $\begin{array}{l}\text { Montero- } \\
\text { Odasso, } \\
2019\end{array}$ & $\begin{array}{l}\text { Chen, } \\
2018\end{array}$ & $\begin{array}{l}\text { Kim, } \\
2017\end{array}$ & $\begin{array}{l}\text { We } \\
20\end{array}$ \\
\hline \multirow[t]{12}{*}{ Reach } & $\begin{array}{l}\text { Described target } \\
\text { population }\end{array}$ & $x$ & $x$ & $x$ & $x$ & $x$ & $x$ & $x$ & $x$ & $x$ & $x$ \\
\hline & $\begin{array}{l}\text { Demographic, } \\
\text { behavioral information } \\
\text { about target } \\
\text { population }\end{array}$ & $x$ & $x$ & $x$ & $x$ & $x$ & $x$ & $x$ & $x$ & $x$ & $x$ \\
\hline & $\begin{array}{l}\text { Method to identify the } \\
\text { target population }\end{array}$ & $x$ & $x$ & $x$ & $x$ & $x$ & $x$ & & $x$ & & $x$ \\
\hline & Recruitment strategies & $x$ & $x$ & & & & & $x$ & $x$ & & $x$ \\
\hline & $\begin{array}{l}\text { Inclusion/exclusion } \\
\text { criteria for individuals }\end{array}$ & $x$ & $x$ & $x$ & $x$ & $x$ & $x$ & $x$ & $x$ & $x$ & $x$ \\
\hline & $\begin{array}{l}\text { Eligible, invited } \\
\text { (exposed to } \\
\text { recruitment) potential } \\
\text { participants }\end{array}$ & $x$ & $x$ & $x$ & $x$ & $x$ & $x$ & $x$ & $x$ & & $x$ \\
\hline & Sample size & $x$ & $x$ & $x$ & $x$ & $x$ & $x$ & $x$ & $x$ & $x$ & $x$ \\
\hline & $\begin{array}{l}\text { Individual participation } \\
\text { rate (sample } \\
\text { size/eligible invited } \\
\text { potential participants) }\end{array}$ & $x$ & $x$ & $x$ & $x$ & $x$ & $x$ & $x$ & $x$ & & $x$ \\
\hline & $\begin{array}{l}\text { Comparisons between } \\
\text { the target population } \\
\text { and the study sample }\end{array}$ & & & & $x$ & & & & & & \\
\hline & $\begin{array}{l}\text { Statistical } \\
\text { comparisons between } \\
\text { the target population } \\
\text { and the study sample }\end{array}$ & & & & $x$ & & & & & & \\
\hline & Cost of recruitment & & & & & & & & & & \\
\hline & $\begin{array}{l}\text { Qualitative methods to } \\
\text { measure reach }\end{array}$ & & & & & & & & & & \\
\hline \multirow[t]{9}{*}{ Effectiveness } & Report of mediators & & & & $x$ & & & & & & \\
\hline & Report of moderators & & & $x$ & $\mathrm{x}$ & $x$ & $x$ & $x$ & $x$ & & \\
\hline & Intent-to-treat & & & $x$ & $x$ & $x$ & & $x$ & $x$ & & $x$ \\
\hline & Imputation procedures & & & $x$ & & $x$ & & & $x$ & & $x$ \\
\hline & $\begin{array}{l}\text { Quality-of-life } \\
\text { measures }\end{array}$ & $x$ & $x$ & $x$ & & & & & & $x$ & \\
\hline & $\begin{array}{l}\text { Unintended } \\
\text { consequences } \\
\text { measures/results }\end{array}$ & $x$ & $x$ & $x$ & & $x$ & $x$ & $x$ & $x$ & & $x$ \\
\hline & $\begin{array}{l}\text { Percent attrition (at } \\
\text { program completion) }\end{array}$ & $\mathrm{x}$ & $x$ & $x$ & $x$ & $x$ & $x$ & $x$ & $x$ & & $x$ \\
\hline & Cost-effectiveness & & & & & & & & & & \\
\hline & $\begin{array}{l}\text { Qualitative methods to } \\
\text { measure } \\
\text { efficacy/effectiveness }\end{array}$ & & & & & & $x$ & & & & \\
\hline \multirow[t]{4}{*}{$\begin{array}{l}\text { Adoption, } \\
\text { setting }\end{array}$} & $\begin{array}{l}\text { Eligible, invited } \\
\text { potential settings }\end{array}$ & & & & & & & & & & \\
\hline & $\begin{array}{l}\text { Number of } \\
\text { participating settings }\end{array}$ & & $x$ & & & $x$ & $x$ & & & $x$ & \\
\hline & $\begin{array}{l}\text { Setting participation } \\
\text { rate }\end{array}$ & & & & & & & & & & \\
\hline & $\begin{array}{l}\text { Description of the } \\
\text { targeted location }\end{array}$ & & & & & & & & & & \\
\hline
\end{tabular}




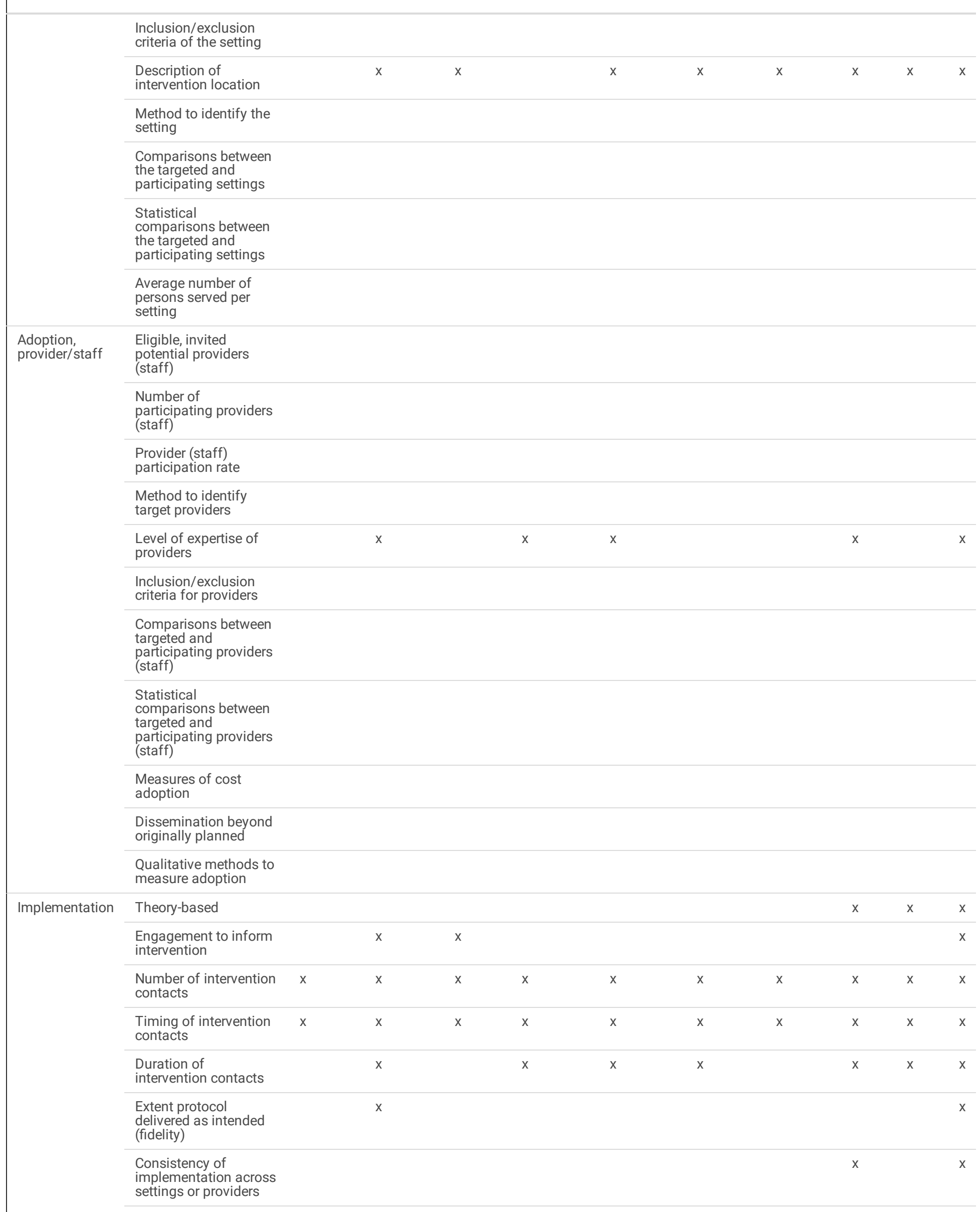




\section{Study}

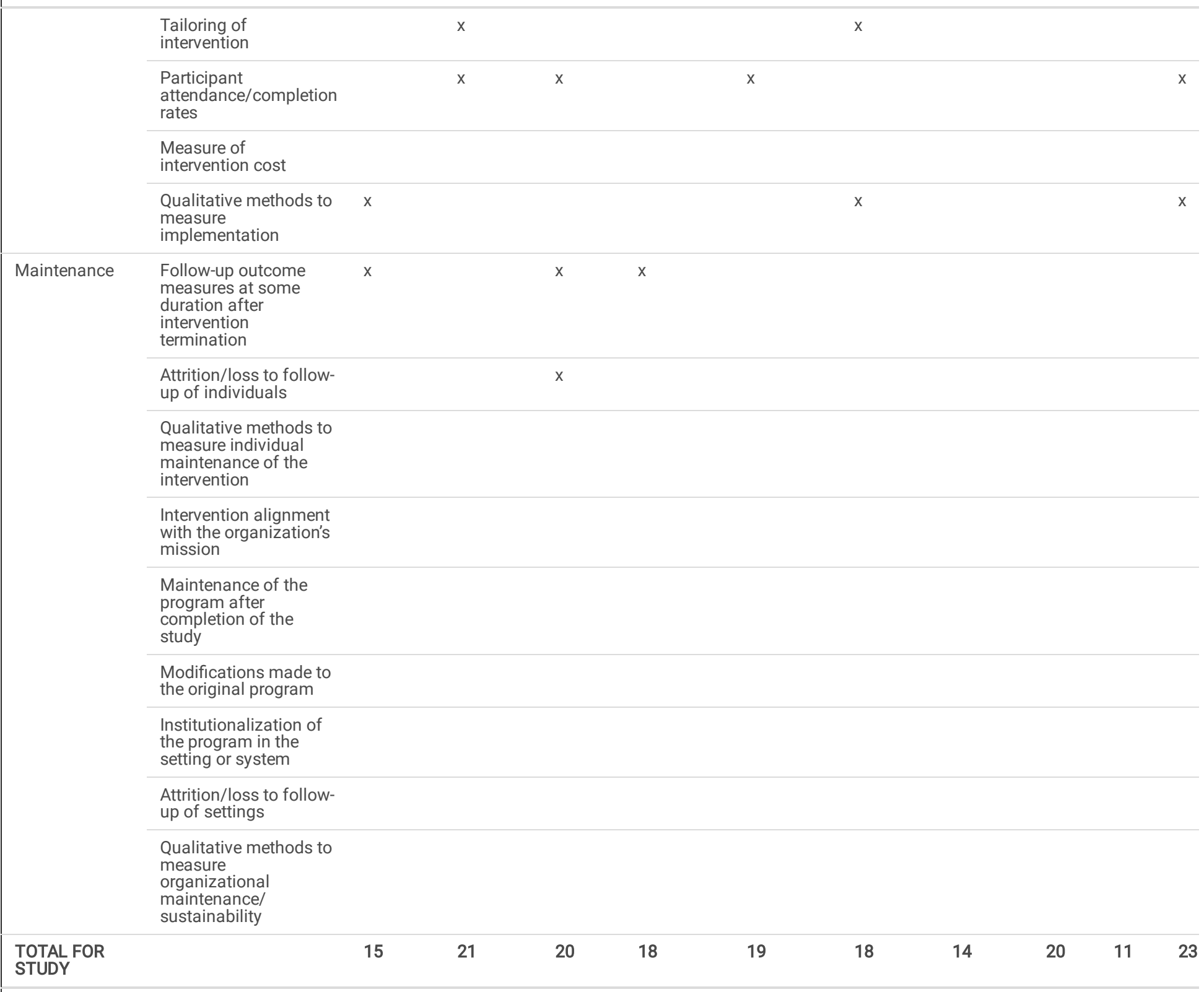

\section{RE-AIM Criteria}

\section{Reach}

Of all the RE-AIM constructs, this was the most thoroughly reported on by the included studies as 7 of the 12 criteria were described by 10 or more of the studies. All studies described the target population, provided demographic and behavioural information about the target population, and the sample size of the study. However, demographic, and behavioural information of the participants was not reported consistently across studies, with studies reporting different sample characteristics. For example, while almost all studies reported on gender and age of their participants, some did not include any information about ethnicity/race $(n=10)$, socioeconomic status $(n=4)$, or chronic diseases $(n=6)$ of their target population. Most of the studies ( $n \geq 10)$ included information about how they identified the target population, inclusion/exclusion criteria, the number of invited participants, and the participation rate. Recruitment strategies were reported by 7 of the included studies. Only one study (34) compared the target population to the study sample, and noted that those who did not participate were older and had a higher disease burden. No studies provided any information on the cost of recruitment or qualitatively measured their reach.

\section{Effectiveness}

Overall, almost all studies $(n=11)$ reported the attrition rate at study completion and 9 studies explicitly reported on any unintended results or adverse events. No study reported any serious adverse events related to the intervention protocol/program. These adverse events included falls, stiffness, dizziness, and mild joint pain, all of which were eased by modifying the program or with continued participation in the intervention. Many studies reported moderators $(n=7)$ and followed intention-to-treat protocols $(n=7)$, but fewer reported on imputation procedures for missing data $(n=5)$ and quality of life outcomes $(n=5)$. No studies addressed cost-effectiveness and only 1 study reported qualitative measures of effectiveness (36) using a Likert scale questionnaire with participants. 


\section{Adoption}

Overall, adoption was very poorly reported by all studies in our review. Nine of the included studies described the intervention location (e.g. home, community centres and research centres) and 4 reported the number of settings where the intervention was delivered. Only 6 studies provided details on the level of expertise required for providers/staff members to implement the intervention. No adoption criteria (e.g. participation rates of settings or providers, inclusion/exclusion criteria of settings or providers, or comparisons across different settings or providers) were reported by any studies.

\section{Implementation}

All studies $(n=12)$ provided information on the number of intervention contacts/visits and 11 studies were explicit in the timing of these intervention visits in regard to when they occurred over the duration of the study. The number of visits and when they occurred varied greatly across included studies and was sometimes poorly described resulting in uncertainty. Only 8 studies provided the duration of intervention visits either within the methods section of the paper or in the results when reporting how long participants took to complete intervention components (i.e., how long they participated in the exercise session). However, no studies reported on how long the measurement or outcome assessments took. Overall, participant attendance or completion rates and qualitative measures of implementation were not well reported by studies. Additionally, only 4 studies specified a theory or framework that informed the development of the intervention and while no studies stated involvement of participants or caregivers in designing the intervention, 4 studies did include caregivers in implementation of intervention components $(5,32,33,37)$. Additionally, four studies purposefully tailored their intervention methods and delivery to the individual participants in their study through individual activity/exercise plans that may have been adjusted for age, illness-related deficits, and/or tailored and specialized programming. Fidelity and consistency of implementation of the intervention was rarely reported on ( $n=2$ for each criteria) and no studies measured the cost of the intervention.

\section{Maintenance}

This construct was poorly reported by all studies in this review. Beyond immediate post intervention measurements, only 3 studies assessed the sustainability of the intervention effects following completion of the intervention $(31,33,34)$. Further, only 1 of these 3 studies reported the loss of participants at this followup time period (33). No studies assessed or reported on any of the other criteria such as maintenance of the program, modifications made to maintain the program, alignment of the intervention with the organization's mission, or any qualitative methods to measure maintenance/sustainability.

\section{Discussion}

This paper builds on a related systematic review which investigated the effectiveness of fall prevention interventions in community-dwelling adults with mild to moderate cognitive impairment. In this paper, we examined the application and reporting of various dimensions of the RE-AIM framework in the studies included in the systematic review to inform future practice-based implementation research of fall prevention initiatives. Overall, we found a general lack of reporting on most RE-AIM criteria. While Reach was the best-reported construct by the included studies, followed by Effectiveness and Implementation, the criteria within the Adoption and Maintenance constructs were rarely mentioned in these studies. In general, the RE-AIM criteria were not applied consistently, with some studies selecting to apply only particular criteria.

The RE-AIM framework was developed to improve the balanced reporting of internal and external validity of behavioural interventions (20). It is thought that the transparent and consistent reporting of interventions may lead to a better understanding of their potential public health impact. However, across different disciplines, reviews that have investigated the use of RE-AIM and its reporting have concluded insufficient and inconsistent information on RE-AIM dimensions which leads to gaps related to who, under what conditions, and how these interventions are successful (29). The need for more detailed reporting of fall prevention interventions for those with cognitive impairment, has been recognized previously (3). There was a lack of reporting by studies on the RE-AIM criteria. This included components such as eligibility criteria, descriptions of interventions, number of participants recruited and randomized, adverse events or harms, among others. Given the adherence to reporting guidelines, these documents and figures could be expanded to increase transparency in reporting external validity (43).

From the included studies in the systematic review, we found that the components reflecting external validity (i.e., Adoption/Diffusion and Maintenance) the most challenging criteria for data extraction. Adoption is specifically segmented at two levels, setting and staff, while Maintenance has criteria that relate to both individual and organizational level. Only one intervention was delivered at multiple sites and it was not designed to test or measure implementation at different sites based on these criteria (32). Not knowing differences in settings and providers makes it difficult to determine what criteria might be needed for a site to successfully deliver the intervention, who in a real-world setting is best suited to deliver the intervention, or what settings might be appropriate for translation. Fall prevention interventions for cognitively impaired older adults also lack implementation details to replicate with fidelity and sustainability. Maintenance was reported on by almost no studies which leaves questions about the sustainability of these interventions, especially given the short duration of the studies. The lack of reporting on these external validity components impacts the ability of researchers and practitioners to translate research results into evidence-based policy and practice and to push the field forward. Without consistent and comprehensive reporting, it is difficult to assess whether the effectiveness of interventions is due to the intervention itself, implementation components. This makes it challenging to inform the science of what and how interventions work and whom they work best with, to improve the development of future interventions.

The results of this review are not surprising given how little attention issues critical to translating research findings to public health often receive when compared with intervention effectiveness. The true impact of interventions is often constrained by barriers to their effective implementation (18). Reviews typically focus on effectiveness of interventions; however, without adequately exploring their implementation, further effective implementation approaches disseminate findings and scale these interventions are limited (44). Although some of the included evidence in the review were feasibility or pilot studies (42\%), they were deficient in reporting on RE-AIM criteria, although three of the five feasibility studies had the three highest number of reported criteria (5, 32 , 37). Ideally, systematic reviews would be able to report on effectiveness of interventions and relate these findings to implementation and external validity 
constructs, which would improve clinical practice and inform healthcare policies $(18,45)$. However, a challenge with this field of research may be the lack of clarity on effective interventions as many of the included studies were small in nature and appeared to be more pragmatic studies. While implementation science is on the rise and increasing in popularity (46), the current state of the science and evidence base regarding fall prevention in community dwelling adults with cognitive impairment has room for improvement.

\section{Limitations}

Although our search was comprehensive, we did not explicitly search grey literature. We did verify our included studies with those of other similar reviews and our results align with previous research in this area. It is also important to recognize that this review is a secondary research question to a systematic review and meta-analysis for which the search was originally intended. This search and inclusion/exclusion criteria for screening was not designed to select studies for implementation trials or RE-AIM fall prevention interventions. Although were not able to relate the reporting of the RE-AIM constructs and criteria with the effectiveness of the interventions, our review is only able to comment on the state of science and research with respect to fall prevention interventions in community-dwelling adults with cognitive impairment.

\section{Implications for Practice and Research}

The gap in the research for reducing falls in those with cognitive impairment and the need for more detailed reporting of these interventions continues to go unmet despite calls by previous research (3), health practitioners and clinicians themselves. Based on the reporting of RE-AIM components in this review, we are unable to make connections to successful intervention components and thus recommendations for practice. Fall prevention research for cognitively impaired adults is not meeting the needs of health practitioners and clinicians and there is little evidence to inform scale-up effective interventions in the community setting. The lack of information regarding external validity greatly limits interpretation and comparisons across studies that are required to fully understand impact and to inform future research efforts $(47,48)$. Once effectiveness is known, more consistent reporting of fall prevention interventions is needed to more successfully translate results into practice.

\section{Conclusions}

Because of the unique risk factors for falls in cognitively impaired adults, it is important that research focuses on effective fall prevention interventions for this high risk group. Researchers, health practitioners and clinicians have highlighted this gap and the need for research to inform evidence-based best practices and recommendations. However, there remains inadequate reporting of fall prevention interventions, especially regarding implementation guidance to health practitioners and clinicians for such interventions. These gaps in reporting of intervention components related to the RE-AIM framework and a lack of high quality studies limits the ability to translate research into practice. More standardized reporting on external validity is needed to determine whether fall prevention interventions or their components can be effectively delivered, in what setting, by whom it can be delivered, and whether it is sustainable in practice.

\section{Declarations}

\section{Ethics approval}

As this study was solely literature based, it was not eligible for institutional ethics approval, and none was sought.

\section{Consent for publication}

Not Applicable

\section{Availability of data and materials}

The main study data is the data extraction materials and quality ratings of included papers, most of which are included in the manuscript tables. Any other supporting data relating to this review is available from the authors.

\section{Competing interests}

The authors declare that they have no competing interests.

\section{Funding}

This research was funded by the Ontario Neurotrauma Foundation (ONF), the McMaster Evidence Review and Synthesis Team (MERST), and the Aging, Community and Health Research Unit (ACHRU) at McMaster University. ONF is funded by the Ontario Ministry of Health. ACHRU is funded by the Max Bell Foundation, Ontario SPOR Support Unit (OSSU), Canadian Institutes of Health Research (CIHR): Catalyst Fund and Pan-Canadian SPOR Network in Primary and Integrated Health Care Innovations (PIHCl), Labarge Optimal Aging Initiative, Labarge Foundation, McMaster Institute for Research on Aging (MIRA), and Diabetes Action Canada. Together, these funds supported the work of Dr. Megan Racey as a post-doctoral fellow. Dr. Diana Sherifali holds the Heather M. Arthur Population Health Research Institute/Hamilton Health Sciences Chair in Interprofessional Health Research, which supported her role in this work. 


\section{Authors' contributions}

All authors were involved in conception and design of the study and approved the protocol; MR, DFL, DS were responsible for overseeing the search of databases and literature and RL handled management of database and deduplication or records. MR, DFL, RL, MJ were involved in the screening of citations; MR, and DFL were responsible for data extraction and analysis of the data. MR, DFL, MUA, HG, SH, JP, RS, LH, DS were involved in interpretation of data. All authors supported in the drafting of the manuscript which was led by MR and all authors supported in revising and formatting of the manuscript. All authors have provided final approval of the version of the manuscript submitted for publication, and all authors agree to be accountable for all aspects of the work.

\section{Acknowledgements}

The authors thank Angela Eady for developing the search strategy.

\section{References}

1. Lipardo DS, Aseron AMC, Kwan MM, Tsang WW. Effect of Exercise and Cognitive Training on Falls and Fall-Related Factors in Older Adults With Mild Cognitive Impairment: A Systematic Review. Archives of Physical Medicine \& Rehabilitation. 2017;98(10):2079-96.

2. Burton E, Cavalheri V, Adams R, Browne CO, Bovery-Spencer P, Fenton AM, et al. Effectiveness of exercise programs to reduce falls in older people with dementia living in the community: a systematic review and meta-analysis. Clin Interv Aging. 2015;10:421-34.

3. Booth V, Logan P, Harwood R, Hood V. Falls prevention interventions in older adults with cognitive impairment: A systematic review of reviews. International Journal of Therapy \& Rehabilitation. 2015;22(6):289-96.

4. Guo JL, Tsai YY, Liao JY, Tu HM, Huang CM. Interventions to reduce the number of falls among older adults with/without cognitive impairment: An exploratory meta-analysis. International Journal of Geriatric Psychiatry. 2014;29(7):661-9.

5. Wesson J, Clemson L, Brodaty H, Lord S, Taylor M, Gitlin L, et al. A feasibility study and pilot randomised trial of a tailored prevention program to reduce falls in older people with mild dementia. BMC geriatrics. 2013;13:89.

6. Winter H, Watt K, Peel NM. Falls prevention interventions for community-dwelling older persons with cognitive impairment: A systematic review. International Psychogeriatrics. 2013;25(2):215-27.

7. Canada PHAo. Seniors' Falls in Canada: Second Report. 2014.

8. Markle-Reid M, Browne G, Gafni A, Roberts J, Weir R, Thabane L, et al. A cross-sectional study of the prevalence, correlates, and costs of falls in older home care clients 'at risk' for falling. Can J Aging. 2010;29(1):119-37.

9. Vassallo M, Mallela SK, Williams A, Kwan J, Allen S, Sharma JC. Fall risk factors in elderly patients with cognitive impairment on rehabilitation wards. Geriatrics and Gerontology International. 2009;9(1):41-6.

10. Fernando E, Fraser M, Hendriksen J, Kim CH, Muir-Hunter SW. Risk Factors Associated with Falls in Older Adults with Dementia: A Systematic Review. Physiotherapy Canada. 2017;69(2):161-73.

11. Beauchet O, Sekhon H, Schott A-M, Rolland Y, Muir-Hunter S, Markle-Reid M, et al. Motoric Cognitive Risk Syndrome and Risk for Falls, Their Recurrence, and Postfall Fractures: Results From a Prospective Observational Population-Based Cohort Study. Journal of the American Medical Directors Association. 2019;20(10):1268-73

12. Whitney J, Close JC, Jackson SH, Lord SR. Understanding risk of falls in people with cognitive impairment living in residential care. J Am Med Dir Assoc. 2012;13(6):535-40.

13. Campbell AJ, Robertson Mc Fau - Gardner MM, Gardner Mm Fau - Norton RN, Norton Rn Fau - Tilyard MW, Tilyard Mw Fau - Buchner DM, Buchner DM. Randomised controlled trial of a general practice programme of home based exercise to prevent falls in elderly women. BMJ. 1997;315(7115):1065-9.

14. Hill KD, Suttanon P, Lin SI, Tsang WWN, Ashari A, Hamid TAA, et al. What works in falls prevention in Asia: a systematic review and meta-analysis of randomized controlled trials. BMC geriatr. 2018;18(1):3.

15. El-Khoury F, Cassou B, Charles MA, Dargent-Molina P. The effect of fall prevention exercise programmes on fall induced injuries in community dwelling older adults: systematic review and meta-analysis of randomised controlled trials. Bmj. 2013;347:f6234.

16. Centre for Clinical Practice at N. National Institute for Health and Care Excellence: Clinical Guidelines. Falls: Assessment and Prevention of Falls in Older People. London: National Institute for Health and Care Excellence (UK) Copyright @ National Institute for Health and Care Excellence, $2013 . ; 2013$.

17. Panel on Prevention of Falls in Older Persons AGS, British Geriatrics S. Summary of the Updated American Geriatrics Society/British Geriatrics Society Clinical Practice Guideline for Prevention of Falls in Older Persons. Journal of the American Geriatrics Society. 2011;59(1):148-57.

18. Child S, Goodwin V, Garside R, Jones-Hughes T, Boddy K, Stein K. Factors influencing the implementation of fall-prevention programmes: a systematic review and synthesis of qualitative studies. Implementation science : IS. 2012;7:91.

19. Sambrook P, Cooper C. Osteoporosis. Lancet. 2006;367(9527):2010-8.

20. Glasgow RE, Vogt TM, Boles SM. Evaluating the public health impact of health promotion interventions: the RE-AIM framework. Am J Public Health. 1999;89(9):1322-7.

21. Shubert TE, Altpeter M, Busby-Whitehead J. Using the RE-AIM framework to translate a research-based falls prevention intervention into a communitybased program: lessons learned. Journal of safety research. 2011;42(6):509-16. 
22. Li F, Harmer P. Protocol for disseminating an evidence-based fall prevention program in community senior centers: evaluation of translatability and public health impact via a single group pre-post study. Implementation Science. 2014;9(1):63.

23. Olij BF, Erasmus V, Barmentloo LM, Burdorf A, Smilde D, Schoon Y, et al. Evaluation of Implementing a Home-Based Fall Prevention Program among Community-Dwelling Older Adults. Int J Environ Res Public Health. 2019;16(6).

24. Racey M, Markle-Reid M, Fitzpatrick-Lewis D, Ali MU, Gagne H, Hunter S, et al. Fall prevention in community-dwelling adults with mild to moderate cognitive impairment: A systematic review and meta-analysis. BMC Geriatrics. 2021;[submitted for review].

25. Hopewell S, Copsey B, Nicolson P, Adedire B, Boniface G, Lamb S. Multifactorial interventions for preventing falls in older people living in the community: a systematic review and meta-analysis of 41 trials and almost 20000 participants. Br J Sports Med. 2020;54(22):1340-50.

26. Institute for Work and Health. Primary, secondary and tertiary prevention Toronto: IWH; 2015 [Available from: https://www.iwh.on.ca/what-researchersmean-by/primary-secondary-and-tertiary-prevention.

27. Markle-Reid MF, Dykeman CS, Reimer HD, Boratto LJ, Goodall CE, McGugan JL. Engaging community organizations in falls prevention for older adults: Moving from research to action. Canadian journal of public health = Revue canadienne de sante publique. 2015;106(4):e189-96.

28. Lamb SE, Becker C, Gillespie LD, Smith JL, Finnegan S, Potter R, et al. Reporting of complex interventions in clinical trials: development of a taxonomy to classify and describe fall-prevention interventions. Trials. 2011;12(1):125

29. Harden SM, Gaglio B, Shoup JA, Kinney KA, Johnson SB, Brito F, et al. Fidelity to and comparative results across behavioral interventions evaluated through the RE-AIM framework: a systematic review. Systematic reviews. 2015;4:155-

30. Hoffmann TC, Glasziou PP, Boutron I, Milne R, Perera R, Moher D, et al. Better reporting of interventions: template for intervention description and replication (TIDieR) checklist and guide. Bmj. 2014;348:g1687.

31. Varriano B, Sulway S, Wetmore C, Dillon W, Misquitta K, Multani N, et al. Vestibular Exercises as a Fall Prevention Strategy in Patients with Cognitive Impairment. Canadian Journal of Neurological Sciences. 2020;47(1):126-30.

32. Goldberg SE, van der Wardt V, Brand A, Burgon C, Bajwa R, Hoare Z, et al. Promoting activity, Independence and stability in early dementia (PrAISED): a, multisite, randomised controlled, feasibility trial. BMC Geriatrics. 2019;19(1):1-12.

33. Padala KP, Padala P, Lensing SY, Dennis RA, Bopp MM, Parkes CM, et al. Home-based Exercise Program Improves Balance and Fear of Falling in Community Dwelling Older Adults with Mild Alzheimer's Disease. Journal of the American Geriatrics Society. 2017;65:S9-S10.

34. Zieschang T, Schwenk M, Becker C, Uhlmann L, Oster P, Hauer K. Falls and Physical Activity in Persons with Mild to Moderate Dementia Participating in an Intensive Motor Training Randomized Controlled Trial. Alzheimer Disease and Associated Disorders. 2017;31(4):307-14.

35. Sungkarat S, Boripuntakul S, Chattipakorn N, Watcharasaksilp K, Lord SR. Effects of Tai Chi on Cognition and Fall Risk in Older Adults with Mild Cognitive Impairment: A Randomized Controlled Trial. Journal of the American Geriatrics Society. 2017;65(4):721-7.

36. Schwenk M, Sabbagh M, Lin I, Morgan P, Grewal GS, Mohler J, et al. Sensor-based balance training with motion feedback in people with mild cognitive impairment. Journal of Rehabilitation Research and Development. 2016;53(6):945-58.

37. Suttanon P, Hill KD, Said CM, Williams SB, Byrne KN, LoGiudice D, et al. Feasibility, safety and preliminary evidence of the effectiveness of a home-based exercise programme for older people with Alzheimer's disease: a pilot randomized controlled trial. Clinical rehabilitation. 2013;27(5):427-38.

38. Hernandez SSS, Coelho FGM, Gobbi S, Stella F. Effects of physical activity on cognitive functions, balance and risk of falls in elderly patients with Alzheimer's dementia. Brazilian Journal of Physical Therapy. 2010;14(1):68-74.

39. Chen YL, Pei YC. Musical dual-task training in patients with mild-to-moderate dementia: A randomized controlled trial. Neuropsychiatric Disease and Treatment. 2018;14:1381-93.

40. Kim KU, Kim SH, Oh HW. The effects of occupation-centered activity program on fall-related factors and quality of life in patients with dementia. J Phys Ther Sci. 2017;29(7):1188-91.

41. Montero-Odasso M, Speechley M, Chertkow H, Sarquis-Adamson Y, Wells J, Borrie M, et al. Donepezil for gait and falls in mild cognitive impairment: A randomized controlled trial. European Journal of Neurology. 2019;26(4):651-9.

42. Schulz KF, Altman DG, Moher D, the CG. CONSORT 2010 Statement: updated guidelines for reporting parallel group randomised trials. BMC Med. 2010;8(1):18.

43. Glasgow RE, Huebschmann AG, Brownson RC. Expanding the CONSORT Figure: Increasing Transparency in Reporting on External Validity. American journal of preventive medicine. 2018;55(3):422-30.

44. Tomoaia-Cotisel A, Scammon DL, Waitzman NJ, Cronholm PF, Halladay JR, Driscoll DL, et al. Context matters: the experience of 14 research teams in systematically reporting contextual factors important for practice change. Ann Fam Med. 2013;11 Suppl 1(Suppl 1):S115-23.

45. Wolfenden L, Foy R, Presseau J, Grimshaw JM, Ivers NM, Powell BJ, et al. Designing and undertaking randomised implementation trials: guide for researchers. BMJ. 2021;372:m3721.

46. Barwick, M., Dubrowski, R., \& Petricca, K. (2020). Knowledge translation: The rise of implementation.Washington, DC: American Institutes for Research.

47. Glasgow RE, Estabrooks PE. Pragmatic Applications of RE-AIM for Health Care Initiatives in Community and Clinical Settings. Preventing chronic disease. 2018;15:E02.

48. Gaglio B, Shoup JA, Glasgow RE. The RE-AIM framework: a systematic review of use over time. Am J Public Health. 2013;103(6):e38-46.

\section{Figures}




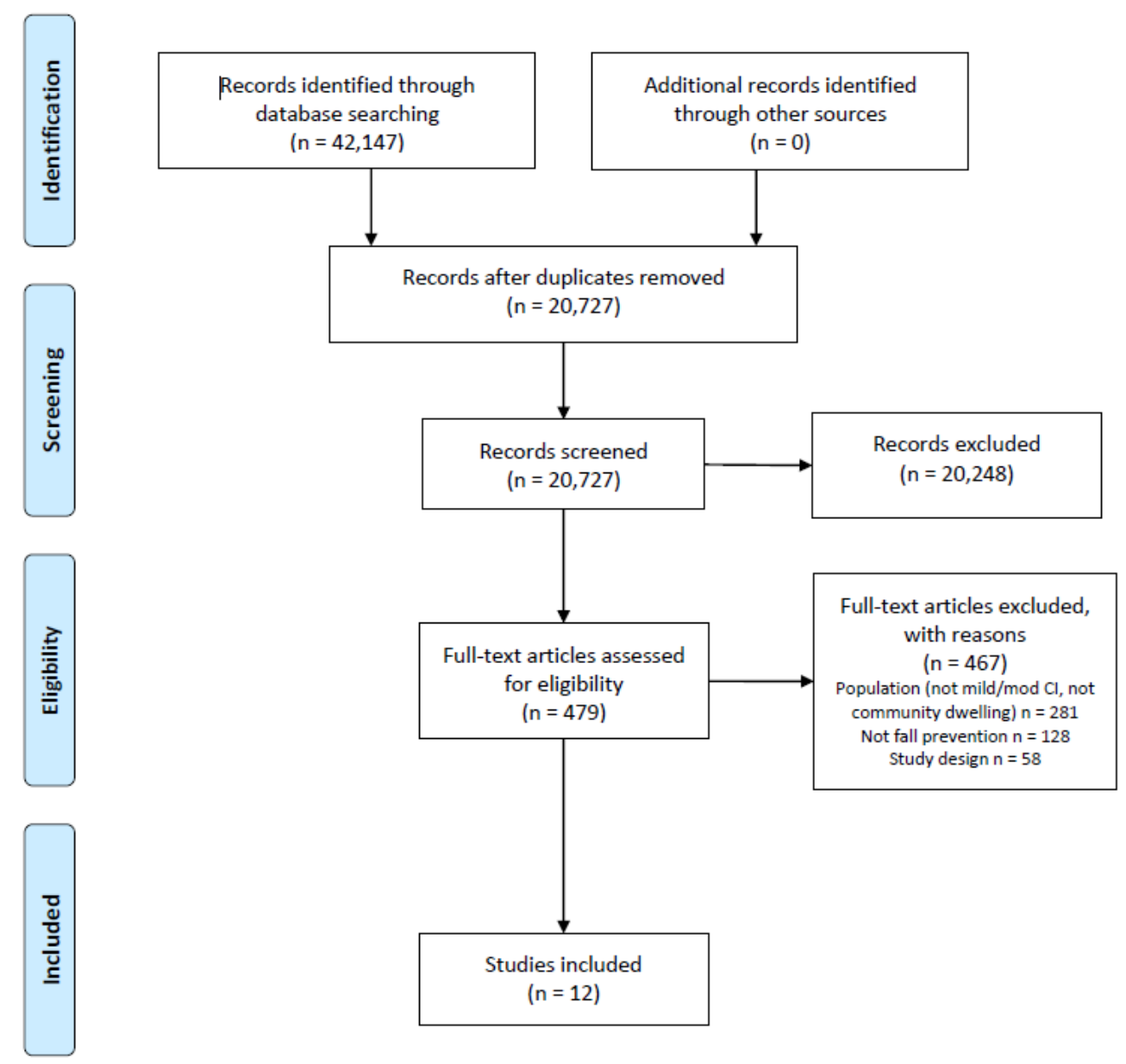

\section{Figure 1}

PRISMA Flowchart

\section{Supplementary Files}

This is a list of supplementary files associated with this preprint. Click to download.

- AdditionalFile1SearchStrategy.pdf

- AdditionalFile2REAIMdataextractioncomponents.doc

- AdditionalFile3CharacteristicsofIncludedStudies.docx

- AdditionalFile4REAIMdataextraction.xlsx 